\title{
Analytical expressions for diffraction-free beams through an opaque disk
}

\section{Qiulin Huang}

\section{Sebastien Coëtmellec}

Fabrice Duval

\section{Anne Louis}

\section{Herve Leblond}

\section{Marc Brunel \\ marc.brunel@coria.fr}

\author{
School of Electronic Engineering, Xidian University, Xi'an, 710071,China \\ Département d'optique, CNRS UMR-6614 CORIA, Av. de l'Université, 76801 Saint-Etienne du Rouvray \\ cedex, France \\ IRSEEM, ESIGELEC, Avenue de l’Universite, 76801 Saint-Etienne du Rouvray, France
}

IRSEEM, ESIGELEC, Avenue de l’Universite, 76801 Saint-Etienne du Rouvray, France

L $\phi$ A, EA4464 , 2 Bd Lavoisier, Université d'Angers, 49045 Angers cedex 01, France

Département d'optique, CNRS UMR-6614 CORIA, Av. de l'Université, 76801 Saint-Etienne du Rouvray cedex, France

We establish analytical expressions that demonstrate that the beam produced after diffraction of a gaussian beam by an opaque disk and collimation by a lens can be approached by a diffraction-free $J_{0}$ Bessel function. We further demonstrate that a similar analytical expression can be established in the case of femtosecond incident pulses. [DOI: 10.2971/jeos.2011.11031]

Keywords: diffraction, Bessel beams, diffraction-free beams

\section{INTRODUCTION}

Diffraction-free beams have attracted much attention since pioneer work of Durnin and co-workers [1]. Different beamshaping techniques exist. Most well-known are based on the use of an annular aperture [1, 2], a computer generatedhologram [3] or an axicon [4]. Although an annular aperture induces many losses, the technique based on this element has recovered much interest in view of recent studies involving sub-wavelength apertures [5]. They could indeed allow the design of systems in sub-wavelength optics.

We have recently proposed another method based on the occultation of an incident beam. We could indeed demonstrate that diffraction of a Gaussian beam by an opaque disk leads to the generation of a diverging Bessel'like beam, that can then be collimated with a lens into a diffraction-compensated beam [6]. The simplicity of the method allows its application directly at the output of a pigtailed laser diode [7]. Numerical developments associated to comparisons with experimental results could validate these results. They showed that the beam diffracted by the opaque disk can be expressed as a sum of approximately 20 Bessel functions of odd orders. Unfortunately, these expressions are so complex that they do not allow to establish any simple expression of the diffractioncompensated beam after the collimating lens. Numerical integration is required to obtain correct simulations. In this case, collimation by the lens is expressed numerically using a phase factor and a Fresnel transform. Any optimization of an experimental set-up requires thus complex numerical integration, in particular to adapt the technique to other domains of wave- length (as in the microwave domain). In addition, with the present formulation, the process appears as a compensation of diffraction between different "orders", and not as the generation of a diffraction-free $J_{0}$ Bessel beam. This method could however present much interest in the next future in view of recent techniques developed in subwavelength optics [5], and combined to intracavity designs [8].

We have thus pursued our study and we present now a simplest formulation that establishes a simplified approached expression of the diffraction'free beam that is generated. After some simplifications that are detailed expressly, the beam is shown to be well approached by a zeroth-order diffractionfree Bessel function. This expression can then be generalized to the case of $100 \mathrm{fs}$ incident pulses. The present paper is organized as follows. In section 2 we establish a new expression of the field diffracted by an opaque disk, assuming an incident Gaussian beam. Results are compared with experimental results and our previous theoretical developments [6]. The domain of validity of this new approach is discussed. In section 3 , collimation by the lens is considered and an expression is established that takes into account the phase factor introduced by the lens, the lens and propagation in free space after the lens. Under assumptions that are discussed, this expression can be much simplified and the beam that is finally obtained is shown to be a non-diffracting zeroth-order Bessel function. Based on these previous developments, we establish then in section 4 an analytical expression of the diffraction-free beam 
produced when the incident Continuous Wave (CW) laser is replaced by a femtosecond laser.

\section{A paraxial solution for the diffraction of a CW beam by an opaque disk}

\subsection{Theoretical development}

Let us consider the set-up presented in figure 1. A Gaussian beam is focused in the vicinity of an opaque disk. The propagation axis is the $z$-axis. $x_{q}, y_{q}$ and $z_{q}$ denote the coordinates in the plane where the opaque disk is located. $x_{q}$ and $y_{q}$ are the transverse coordinates. $x, y$ and $z$ are the coordinates in the plane where the diffracted electric field is calculated $\left(z>z_{q}\right)$. The scalar field of the Gaussian beam over the plane where the opaque disk is located is written as

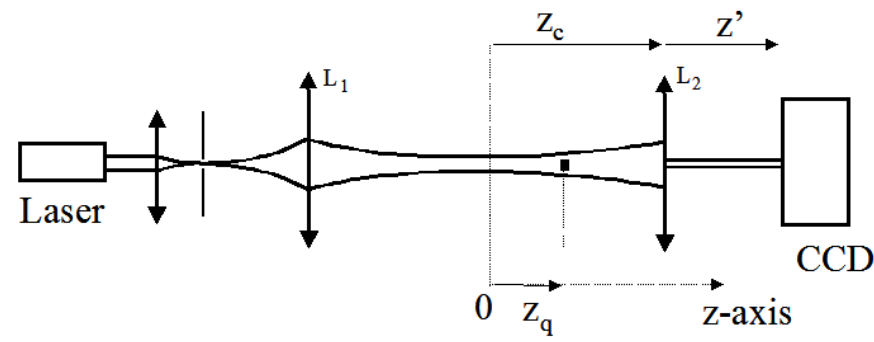

FIG. 1 Experimental set-up. The opaque disk is on the focus point of the lens $L_{2}$

$$
u\left(x_{q}, y_{q}, z_{q}\right)=\exp \left(-\frac{x_{q}^{2}+y_{q}^{2}}{w\left(z_{q}\right)^{2}}\right) \exp \left(-i k \frac{x_{q}^{2}+y_{q}^{2}}{2 R\left(z_{q}\right)}\right)
$$

where $k=2 \pi / \lambda$ represents the wave number and $\lambda$ is the wavelength. The waist and the radius of curvature along the propagation axis $z$ are given by:

$$
\omega(z)=\sqrt{\omega_{0}^{2}\left(1+\left[\frac{z}{z_{0}}\right]^{2}\right)}
$$

and

$$
R(z)=-z\left(1+\left[\frac{z_{0}}{z}\right]^{2}\right)
$$

where $z_{0}=\pi \omega_{0}^{2} / \lambda$ represents the Rayleigh length of the beam, $\omega_{0}$ its width at the waist. The origin position $z=0$ corresponds to the position of the beam waist between the two lens $L_{1}$ and $L_{2}$ (see figure 1 ). Some previous developments have been done to describe this phenomenon and they are detailed in reference [6]. We present here a new formulation that leads to simplified approached expressions. According to the paraxial approximation, we get the following formula that represents the distribution of scalar field [9],

$$
\begin{aligned}
u(x, y, z)=\iint_{\sigma} & \frac{1}{i \lambda\left(z-z_{q}\right)} \\
& \times \exp \left(-\frac{x_{q}^{2}+y_{q}^{2}}{w\left(z_{q}\right)^{2}}\right) \exp \left(-i k \frac{x_{q}^{2}+y_{q}^{2}}{2 R\left(z_{q}\right)}\right) \\
& \times \exp \left(i k\left(z-z_{q}\right)\right. \\
& \left.+\frac{\left(x-x_{q}\right)^{2}+\left(y-y_{q}\right)^{2}}{2\left(z-z_{q}\right)}\right) d x_{q} d y_{q},
\end{aligned}
$$

The integral is calculated on the plane where the opaque disk is located. Let us note $x_{q}=\rho_{q} \cos \varphi, y_{q}=\rho_{q} \sin \varphi$ and $x=\rho \cos \theta, y=\rho \sin \theta$. Then:

$$
\begin{aligned}
u(x, y, z)= & \frac{\exp \left(i k\left(z-z_{q}\right)\right)}{i \lambda\left(z-z_{q}\right)} \int_{D / 2}^{+\infty} \int_{0}^{2 \pi} \exp \left(-\frac{\rho_{q}^{2}}{w\left(z_{q}\right)^{2}}\right) \\
& \times \exp \left(-i k \frac{\rho_{q}^{2}}{2 R\left(z_{q}\right)}\right) \\
& \times \exp \left(i k \frac{\rho^{2}+\rho_{q}^{2}-2 \rho \rho_{q} \cos (\varphi-\theta)}{2\left(z-z_{q}\right)}\right) \\
& \times \rho_{q} d \rho_{q} d \varphi
\end{aligned}
$$

which gives noting $\rho_{q}=D \gamma / 2$ with $\gamma \in[1,+\infty[$ :

$$
\begin{aligned}
u(x, y, z)= & \frac{\exp \left(i k\left(z-z_{q}\right)\right)}{i \lambda\left(z-z_{q}\right)} \exp \left(i k \frac{\rho^{2}}{2\left(z-z_{q}\right)}\right) \frac{D^{2}}{4} \\
& \times \int_{1}^{+\infty} \int_{0}^{2 \pi} \exp \left(-a(z) \gamma^{2}\right) \\
& \times \exp \left(-i \frac{k \rho D \gamma}{2\left(z-z_{q}\right)} \cos (\varphi-\theta)\right) \gamma d \gamma d \varphi
\end{aligned}
$$

with

$$
a(z)=\frac{D^{2}}{4}\left(\frac{1}{w\left(z_{q}\right)^{2}}-i k\left(\frac{1}{2\left(z-z_{q}\right)}-\frac{1}{2 R\left(z_{q}\right)}\right)\right)
$$

Thus:

$$
\begin{aligned}
u(x, y, z)= & -\frac{i \pi D^{2} \exp \left(i k\left(z-z_{q}\right)\right)}{2 \lambda\left(z-z_{q}\right)} \exp \left(i k \frac{\rho^{2}}{2\left(z-z_{q}\right)}\right) \\
& \times \int_{1}^{+\infty} \exp \left(-a(z) \gamma^{2}\right) J_{0}\left(\frac{k \rho D \gamma}{2\left(z-z_{q}\right)}\right) \gamma d \gamma
\end{aligned}
$$

Here, we use the method of integration by parts and the following property of Bessel functions:

$$
\frac{d}{w d w}\left(\frac{J_{v}(w)}{w^{v}}\right)=-\frac{J_{v+1}(w)}{w^{v+1}}
$$


the integral in equation (8) can be represented as the series of Bessel functions, i.e.:

$$
\begin{aligned}
& \int_{1}^{+\infty} \exp \left(-a(z) \gamma^{2}\right) J_{0}\left(\frac{k \rho D \gamma}{2\left(z-z_{q}\right)}\right) \gamma d \gamma \\
= & \sum_{m=0}^{+\infty}(-1)^{m}\left(\frac{1}{2 a(z)}\right)^{m+1}\left(\frac{k \rho D}{2\left(z-z_{q}\right)}\right)^{m} \\
& \times J_{m}\left(\frac{k \rho D}{2\left(z-z_{q}\right)}\right) \exp (-a(z))
\end{aligned}
$$

Using an asymptotic expression of $J_{m}(X)$ for large values of $m$, it can be shown that the series converges. However, for moderate values of $m$ (due to the truncation of the series which is done numerically in practice), $J_{m}(X)$ decreases quite slowly with $m$, and hence the accurateness of the series (10) is measured by the convergence condition for the series :

$$
\sum_{m=0}^{+\infty}\left(-\frac{k \rho D}{4 a(z)\left(z-z_{q}\right)}\right)^{m}
$$

i.e. by

$$
k \rho D /\left(4|a(z)|\left(z-z_{q}\right)\right)<1
$$

which gives:

$$
\rho<4|a(z)|\left(z-z_{q}\right) /(k D)
$$

Then the following paraxial solution is obtained:

$$
\begin{aligned}
u(x, y, z)= & -\frac{i \pi D^{2} \exp \left(i k\left(z-z_{q}\right)\right)}{2 \lambda\left(z-z_{q}\right)} \exp \left(i k \frac{\rho^{2}}{2\left(z-z_{q}\right)}\right) \\
& \times \exp (-a(z)) \sum_{m=0}^{+\infty}(-1)^{m}\left(\frac{1}{2 a(z)}\right)^{m+1} \\
& \times\left(\frac{k \rho D}{2\left(z-z_{q}\right)}\right)^{m} J_{m}\left(\frac{k \rho D}{2\left(z-z_{q}\right)}\right)
\end{aligned}
$$

\subsection{First-order approximation}

Equation (14) can be simplified since it can be well represented only by the zeroth-order Bessel function in paraxial approximation. So, we get

$$
\begin{aligned}
u\left(x, y, z_{c}\right)= & -\frac{i \pi D^{2} \exp \left(i k\left(z_{c}-z_{q}\right)\right)}{2 \lambda\left(z_{c}-z_{q}\right)} \\
& \times \exp \left(i k \frac{\rho^{2}}{2\left(z_{c}-z_{q}\right)}\right) \exp \left(-a\left(z_{c}\right)\right) \\
& \times \frac{1}{2 a\left(z_{c}\right)} J_{0}\left(\frac{k \rho D}{2\left(z_{c}-z_{q}\right)}\right)
\end{aligned}
$$

with $\rho^{2}=\sqrt{x^{2}+y^{2}}$. Figure 2 shows the comparison between an experimental profile, and the previous expansion considering only the coefficient $\mathrm{m}=0$ (first-order approximation). For these experimental results, the laser source is a CW He-Ne laser emitting at $632.8 \mathrm{~nm}$. Beam filtering is ensured by focusing the laser beam with a microscope objective through a pinhole. The filtered diverging beam, which is a quasi-gaussian beam, is then focused with a $2 \mathrm{~cm}$-large lens $L_{1}$ whose focal length is $10 \mathrm{~cm}$. Focusing occurs $15 \mathrm{~cm}$ after the lens $L_{1}$. The diameter of the focus point is $70 \mu \mathrm{m}$ at 1 /e. An opaque disk whose diameter is $300 \mu \mathrm{m}$ is positioned just before the focus point. It is well centered on the optical axis of the experiment, but $3.5 \mathrm{~mm}$ behind the focus point. The diffracted pattern is the one observed with a CCD camera positioned $10.5 \mathrm{~cm}$ after the opaque disk. We can see that the first-order approximation using only a $J_{0}$ Bessel function allows a good fit of experimental results.

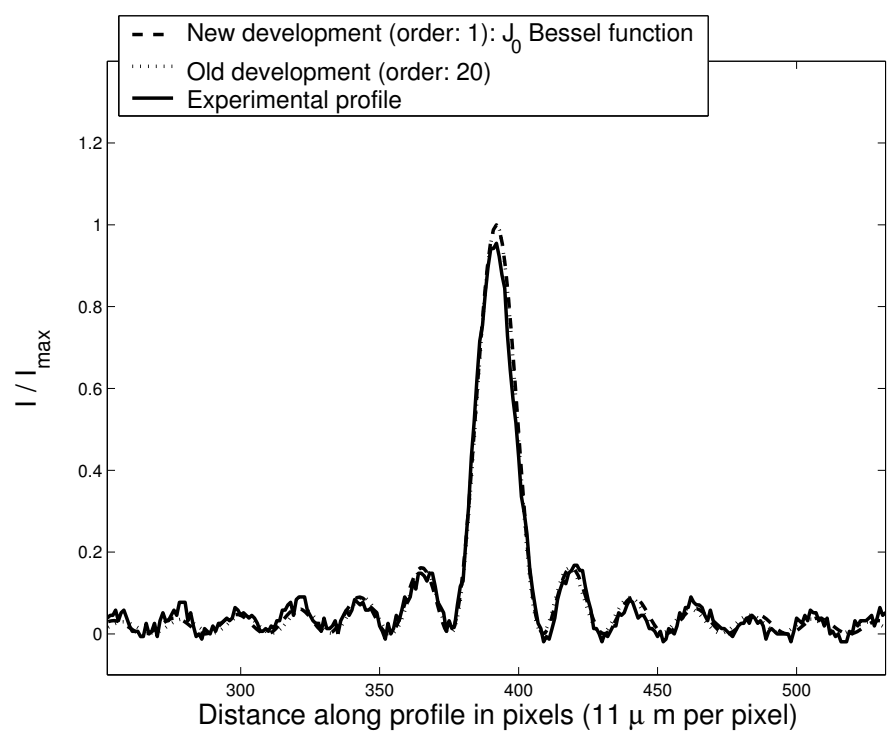

FIG. 2 Comparison of an experimental profile and the first order approximation using a $J_{0}$ Bessel function.

Figure 3 shows some comparisons using our old development with 20 Bessel functions of odd orders (see reference [6]), and the new development with 1, 2 or 8 functions. The parameters are those of the experiment. In the case of the $J_{0}$ approximation (limitation of the new expansion to the first order), the profiles obtained are in good accordance for the central peak and the 3 first concentric rings. Some slight difference concerning the value of the other ring's radius occurs but it does never exceed $2.2 \%$. The new expansion with 2 Bessel functions agrees well with old expansion for the central peak and the 4 first concentric rings. The intensity of the following rings is then slightly overestimated while there is not any error concerning their radius. The new expansion over 8 Bessel functions fits very well the old expansion for the central peak and the 9 first rings. The figure is limited to the domain of accuracy of the new truncated expansion expressed previously $\rho<4|a(z)|\left(z-z_{q}\right) /(k D)$. In this case, the limit is $\rho<2.5 \mathrm{~mm}$. After this limit, the new truncated expansions diverge (except the expansion limited to the sole $J_{0}$ Bessel function). 


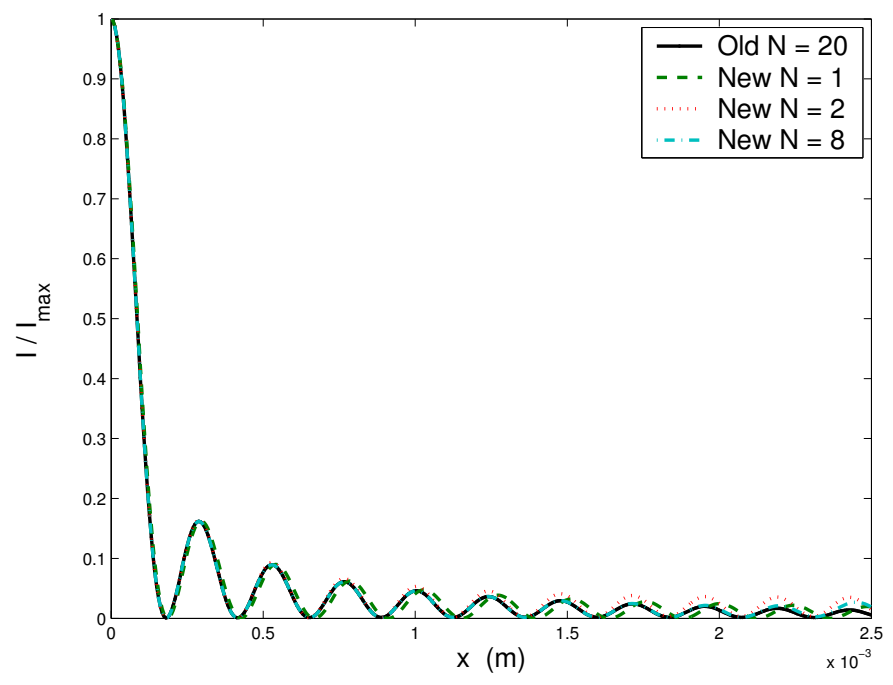

FIG. 3 Comparison of old expansion using 20 Bessel functions of odd orders, and different orders of the new expansion

\section{Collimation of the beam with a lens}

\subsection{Theoretical development}

Let us now consider focusing by the lens as detailed in figure 1. On the plane $z=z_{c}$ where the collimating lens is located, the scalar field can be well represented only by the zerothorder Bessel function in paraxial approximation. The amplitude of the scalar field on the plane $z^{\prime}$ after the collimating lens $L_{2}$ can be written as

$$
\begin{aligned}
u\left(x^{\prime}, y^{\prime}, z^{\prime}\right)= & \frac{\exp \left(i k\left(z^{\prime}-z_{c}\right)\right.}{i \lambda\left(z^{\prime}-z_{c}\right)} \iint_{L_{2}} u\left(x, y, z_{\mathcal{c}}\right) \\
& \times \exp \left(-i k \frac{x^{2}+y^{2}}{2 f_{L_{2}}}\right) \\
& \times \exp \left(i k \frac{\left(x-x^{\prime}\right)^{2}+\left(y-y^{\prime}\right)^{2}}{2\left(z^{\prime}-z_{c}\right)}\right) d x d y
\end{aligned}
$$

where $u\left(x, y, z_{c}\right)$ is given by the first-order approximation of relation (15). It gives:

$$
\begin{aligned}
u\left(x^{\prime}, y^{\prime}, z^{\prime}\right)= & -\frac{\exp \left(i k\left(z^{\prime}-z_{c}\right)\right.}{i \lambda\left(z^{\prime}-z_{c}\right)} \frac{i \pi D^{2} \exp \left(i k\left(z_{c}-z_{q}\right)\right)}{2 \lambda\left(z_{c}-z_{q}\right)} \\
& \times \exp \left(-a\left(z_{c}\right)\right) \frac{1}{2 a\left(z_{c}\right)} \\
& \times \iint_{L_{2}} \exp \left(i k \frac{\rho^{2}}{2\left(z_{c}-z_{q}\right)}\right) \\
& \times J_{0}\left(\frac{k \rho D}{2\left(z_{c}-z_{q}\right)}\right) \exp \left(-i k \frac{\rho^{2}}{2 f_{L_{2}}}\right) \\
& \times \exp \left(i k \frac{\rho^{2}+\rho^{\prime 2}-2 \rho \rho^{\prime} \cos (\varphi-\theta)}{2\left(z^{\prime}-z_{c}\right)}\right) \\
& \times \rho d \rho d \varphi
\end{aligned}
$$

In the above formula: $x^{\prime}=\rho^{\prime} \cos \theta, y^{\prime}=\rho^{\prime} \sin \theta$. If the opaque disk is just located on the focus point of the lens $L_{2}$, that is $f_{L_{2}}=z_{\mathcal{c}}-z_{q}, u\left(x^{\prime}, y^{\prime}, z^{\prime}\right)$ can be written as follows

$$
\begin{aligned}
& u\left(x^{\prime}, y^{\prime}, z^{\prime}\right)= \\
& -\frac{\pi D^{2} \exp \left(i k\left(z^{\prime}-z_{q}+\rho^{\prime 2} /\left(2\left(z^{\prime}-z_{c}\right)\right)\right)-a\left(z_{c}\right)\right)}{4 \lambda^{2} a\left(z_{c}\right)\left(z^{\prime}-z_{c}\right)\left(z_{c}-z_{q}\right)} \\
& \times \iint_{L_{2}} \exp \left(i k \frac{\rho^{2}}{2\left(z^{\prime}-z_{c}\right)}\right) J_{0}\left(\frac{k \rho D}{2\left(z_{c}-z_{q}\right)}\right) \\
& \times \exp \left(-i k \frac{\rho \rho^{\prime} \cos (\varphi-\theta)}{z^{\prime}-z_{c}}\right) \rho d \rho d \varphi
\end{aligned}
$$

\subsection{Infinite lens approximation}

The previous expression (18) leads to a very simple result when the radius of the collimating lens $L_{2}$ tends to infinity. After integration over variable $\varphi$ and according to references [10] which give the following expression :

$$
\begin{aligned}
\int_{0}^{+\infty} & \exp \left(i \frac{k \rho^{2}}{2\left(z^{\prime}-z_{c}\right)}\right) J_{0}\left(\frac{k \rho D}{2\left(z_{c}-z_{q}\right)}\right) \\
& \times J_{0}\left(\frac{k \rho \rho^{\prime}}{z^{\prime}-z_{c}}\right) \rho d \rho \\
= & \frac{i\left(z^{\prime}-z_{c}\right) \exp \left(-i \frac{k\left(z^{\prime}-z_{c}\right) D^{2}}{8\left(z_{\mathcal{c}}-z_{q}\right)^{2}}\right) J_{0}\left(\frac{k \rho^{\prime} D}{2\left(z_{c}-z_{q}\right)}\right)}{k \exp \left(i \frac{k \rho^{\prime 2}}{2\left(z^{\prime}-z_{\mathcal{c}}\right)}\right)}
\end{aligned}
$$

we obtain:

$$
\begin{aligned}
u\left(x^{\prime}, y^{\prime}, z^{\prime}\right)= & -\frac{i \pi D^{2} \exp \left(i k\left(z^{\prime}-z_{q}\right)-a\left(z_{c}\right)\right)}{4 \lambda a\left(z_{c}\right)\left(z_{c}-z_{q}\right)} \\
& \times \exp \left(-i \frac{k\left(z^{\prime}-z_{c}\right) D^{2}}{8\left(z_{c}-z_{q}\right)^{2}}\right) J_{0}\left(\frac{k \rho^{\prime} D}{2\left(z_{c}-z_{q}\right)}\right)
\end{aligned}
$$

The beam generated after the lens can thus be reasonably expressed as a non-diffracting $J_{0}$ Bessel beam under a few approximations. Figure 4 shows some comparison between the beam patterns predicted $30 \mathrm{~cm}$ after the collimating lens (Fig 4a) using the old expansion over 20 Bessel functions of odd orders accompanied by phase factor of the lens and Fresnel transform after the collimating lens (see reference [6]), and the pattern predicted using the first-order $J_{0}$ Bessel function approximation (Fig 4b). Figure 5 shows then a comparison between an experimental profile recorded $1 \mathrm{~m}$ after the collimating lens. Other parameters are the opaque disk diameter $D=300 \mu \mathrm{m}, z_{q}=3.5 \mathrm{~mm}$, and $f_{L_{2}}=25 \mathrm{~mm}$. Although the fit is not perfect (which is not so surprising in view of the assumptions that have been already done), the $J_{0}$ expression appears as a relatively good approximation. The expression derived presently is of course not as precise as our previous rigorous derivations [6]. It represents however an important simplification: it allows indeed the simple design of any system in any domain of wavelength, without need of long numerical procedures. The rigorous developments of reference [6] remain however interesting for the proper adjustment of a system, in the last stage of its realization. 
(a)
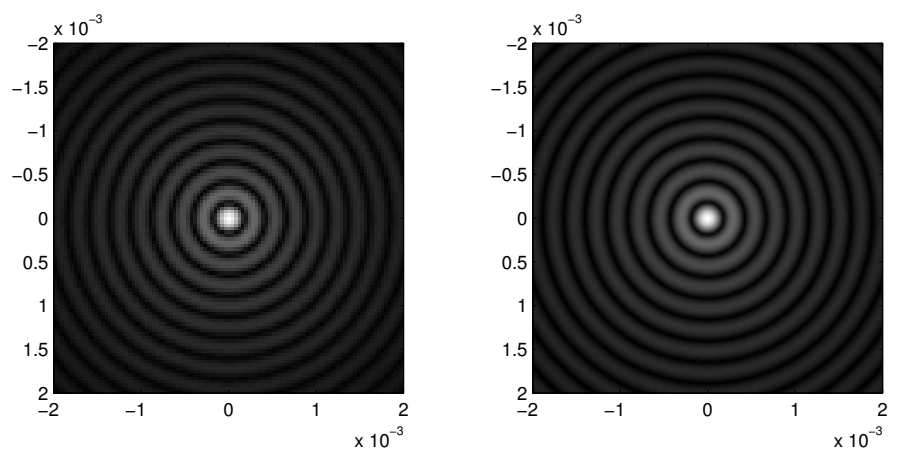

FIG. 4 Comparison of old theoretical development and $J_{0}$ approximation

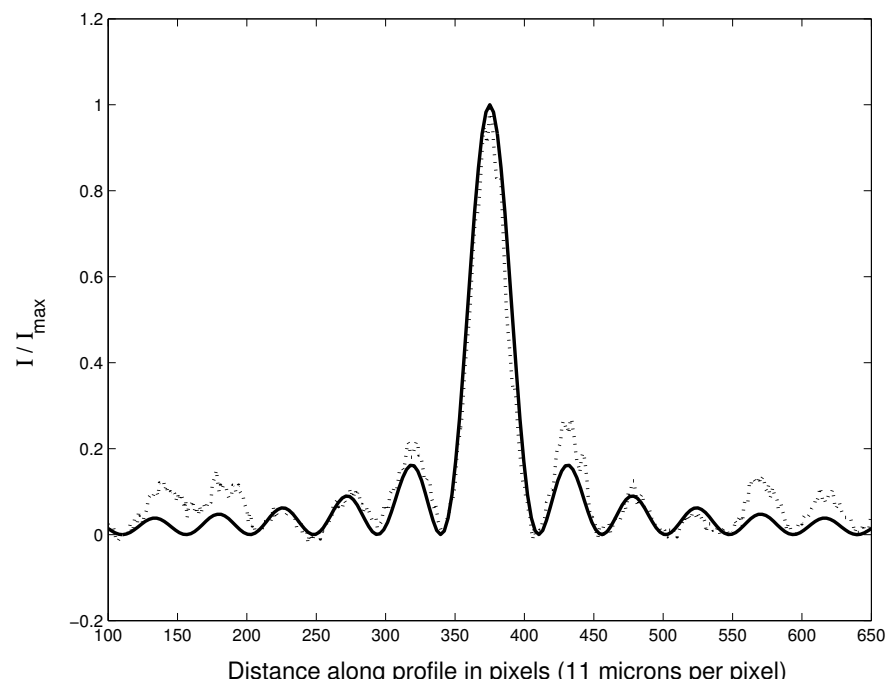

FIG. 5 Comparison of an experimental profile with a first order approximation using a $J_{0}$ function. (a)

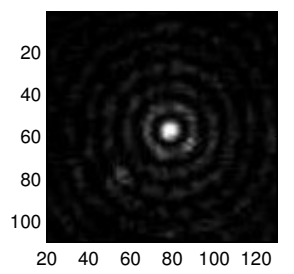

(b)

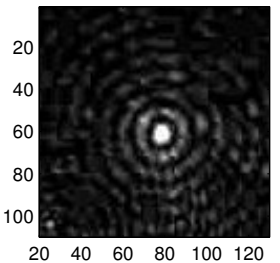

(c)

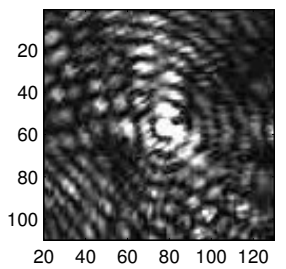

Pixels (4.6 microns per pixel)

FIG. 6 Beam profiles recorded $5 \mathrm{~cm}, 25 \mathrm{~cm}$ and $55 \mathrm{~cm}$ after the lens $L_{2}$

Figure 6 shows an example of non-diffracting beam realization. In this case, the different parameters are $\lambda=632.8 \mathrm{~nm}$, $D=1 \mathrm{~mm}, f_{L_{2}}=6.35 \mathrm{~cm}, R=2.5 \mathrm{~cm}$. The figure presents the patterns recorded $5 \mathrm{~cm}, 25 \mathrm{~cm}$ and $55 \mathrm{~cm}$ after the lens $L_{2}$. In figures b and c, the camera is deliberately saturated to enhance the defaults that appear progressively in the diffraction patterns: when the propagation distance reaches $z=55 \mathrm{~cm}$, parasitic fringes destroy the central peak. With these conditions (a relatively big value of $D$ and small value of $f_{L_{2}}$ ), the dimension of the central peak is relatively small. The experiment has thus been carried out with a specific camera whose pixel size is as small as $4.6 \mu \mathrm{m}$. The diameter of the first dark ring surrounding the central intense peak is approximately 13 pixels for all three recorded images, i.e. $60 \pm 5 \mu \mathrm{m}$. For comparison, the previous approached relation using the sole $J_{0}$ Bessel function gives a diameter of the first dark ring equal to $62.4 \mu \mathrm{m}$. The waist of the central peak (beam radius at $\left.1 / e^{2}\right)$ is $16 \mu \mathrm{m} \pm 5 \mu \mathrm{m}$ in all three cases. Let us notice that the Rayleigh length $z_{r}=\pi \omega_{0}^{2} / \lambda$ of a gaussian beam whose waist dimension would be $\omega_{0}=16 \mu \mathrm{m}$ is $z_{r}=1.2 \mathrm{~mm}$. The $L_{\max }$ that we obtain here $(55 \mathrm{~cm})$ is thus 460 times the Rayleigh length of an equivalent gaussian beam.

\section{Femtosecond diffraction-free bessel beam}

Let us now consider the case of femtosecond pulses. As the femtosecond pulses exhibit a large spectrum, the previous model cannot be used anymore. Diffraction of ultrashort femtosecond pulses has received much attention in the last years and different effects have been evidenced such as spectrum red-shift, pulse time broadening and delay increasing towards the beam periphery, spectrum blue-shift along the propagation direction (see for example [11]). However, these effects become really significant when considering few-cycle pulses. As our pulse duration is relatively long (100 fs), these effects remain low. We consider here ultrashort pulsed beams with constant waist width, extensively used in the literature $[11,12,13]$, under the paraxial approximation. Diffraction is a linear, isotropic and homogeneous effect. The propagation of light can be described by a combination of Fresnel diffraction (for each spectral component) and a temporal filter (for proper superposition of monochromatic components) [14, 15]. The pulse energy does not exceed a few tens of picojoules, such that there is no additional nonlinear effect in the substrate of the diffractive element.

In the temporal Fourier domain, we can write:

$$
\widetilde{E}(\mathbf{r}, \omega)=\widetilde{U}(\mathbf{r}, \omega) \widetilde{G}(\omega)
$$

where $\widetilde{E}(\mathbf{r}, \omega)$ is the temporal Fourier transform of the diffracted electric field $E(\mathbf{r}, t)$ at position $\mathbf{r}$, at time $t$. The function $\widetilde{G}(\omega)$ is the spectrum of the incident pulses. We will consider the following spectrum:

$$
|\widetilde{G}(\omega)|^{2}=\exp \left(-\left(\frac{\omega-\omega_{0}}{\Delta \omega}\right)^{2}\right)
$$

This expression is of course just an approximation. The definition of a universal spectrum for femtosecond pulses is not possible: let us for example cite the wide range of regimes existing in the case of femtosecond fiber-oscillators (solitonic, gaussian or parabolic pulses...). Simulations show that the shape of the spectrum is not a fundamental parameter in our case. The main parameter is indeed the spectrum width (here $\Delta \omega$ ). $\widetilde{U}(\mathbf{r}, \omega)$ is the complex amplitude diffracted by the disk in the case of a perfectly monochromatic plane wave of frequency $\omega$. In the absence of the collimating lens $L_{2}$, its expression, at distance $z$ after the disk on point $\mathbf{r}=(\rho, z)$ (in cylindrical coordinates), is directly deduced from the analysis developed previously. 
By using the Parseval theorem, we can calculate the diffraction intensity with the following formula:

$$
\begin{aligned}
I(\rho, z)= & \frac{C}{16\left(z-z_{q}\right)^{2}} \exp \left(-\frac{D^{2}}{2 w\left(z_{q}\right)^{2}}\right) \\
& \times \int_{-\infty}^{+\infty}|\widetilde{U}(\mathbf{r}, \omega)|^{2}|\widetilde{G}(\omega)|^{2} d \omega
\end{aligned}
$$

where $\mathrm{C}$ is a constant of normalization. Previous relation gives the expression:

$$
\begin{aligned}
I(\rho, z)= & \frac{C w\left(z_{q}\right)^{4}}{16\left(z-z_{q}\right)^{2}} \exp \left(-\frac{D^{2}}{2 w\left(z_{q}\right)^{2}}\right) \\
& \times \int_{-\infty}^{+\infty} \frac{1}{1+\tau^{2} \omega^{2}} \exp \left(-\left(\frac{\omega-\omega_{0}}{\Delta \omega}\right)^{2}\right) \\
& \times J_{0}^{2}(\alpha \omega) d \omega
\end{aligned}
$$

with $\tau=\frac{w\left(z_{q}\right)^{2}}{2 c}\left(\frac{1}{z-z_{q}}-\frac{1}{R\left(z_{q}\right)}\right)$ and $\alpha=\frac{\rho D}{2 c\left(z-z_{q}\right)}$.

To best of our knowledge, this integral cannot be computed analytically. The typical values of the different parameters are $\alpha \sim 10^{-14} s, \tau \sim 10^{-16} s, \omega_{0} \sim 3000 \mathrm{THz}, \Delta \omega \sim 30 \mathrm{THz}$. Introducing the new variable $x=\left(\omega-\omega_{0}\right) / \Delta \omega$, it is possible to make a Taylor-type limited development versus parameter $\Delta \omega$ that is much smaller than $\omega_{0}$ of function

$$
\frac{1}{1+\tau^{2}\left(\omega_{0}+\Delta \omega x\right)^{2}} \exp \left(-x^{2}\right) J_{0}^{2}\left(\alpha\left(\omega_{0}+\Delta \omega x\right)\right)
$$

Integration is then possible. The fourth-order development gives for example the following relation:

$$
\begin{aligned}
& \int_{-\infty}^{+\infty} \frac{\Delta \omega}{1+\tau^{2}\left(\omega_{0}+\Delta \omega x\right)^{2}} \exp \left(-x^{2}\right) \\
& \times J_{0}^{2}\left(\alpha\left(\omega_{0}+\Delta \omega x\right)\right) d x \\
= & \frac{\Delta \omega \sqrt{\pi} J_{0}^{2}\left(\alpha \omega_{0}\right)}{1+\tau^{2} \omega_{0}^{2}}+\frac{(\Delta \omega)^{3} \sqrt{\pi}}{2 \omega_{0}\left(1+\tau^{2} \omega_{0}^{2}\right)^{3}} \\
& \times\left(-\omega_{0}\left(\tau^{2}-3 \tau^{4} \omega_{0}^{2}+\alpha^{2}\left(1+\tau^{2} \omega_{0}^{2}\right)^{2}\right) J_{0}\left(\alpha \omega_{0}\right)^{2}\right) \\
& +\frac{(\Delta \omega)^{3} \sqrt{\pi}}{2 \omega_{0}\left(1+\tau^{2} \omega_{0}^{2}\right)^{3}} \\
& \times\left(\alpha\left(1+6 \tau^{2} \omega_{0}^{2}+5 \tau^{4} \omega_{0}^{4}\right) J_{0}\left(\alpha \omega_{0}\right) J_{1}\left(\alpha \omega_{0}\right)\right. \\
& \left.+\alpha^{2} \omega_{0}\left(1+\tau^{2} \omega_{0}^{2}\right)^{2} J_{1}\left(\alpha \omega_{0}\right)^{2}\right)+O(\Delta \omega)^{5}
\end{aligned}
$$

Figure 7 shows the intensity profiles predicted using firstorder, third-order and fifth-order Taylor-type developments. It appears clearly that the first-order development is sufficient to simulate the intensity profile diffracted by the opaque disk. In other words, in the case of $100 \mathrm{fs}$ pulses, the pulse spectrum is not sufficiently large to affect significantly the paraxial Bessel beam. All developments give similar results in the paraxial domain where the $J_{0}$ Bessel beam approximation was justified.

Assuming now an infinite dimension for the collimating lens, it is possible to express the electric field after the lens $L_{2}$ (in the

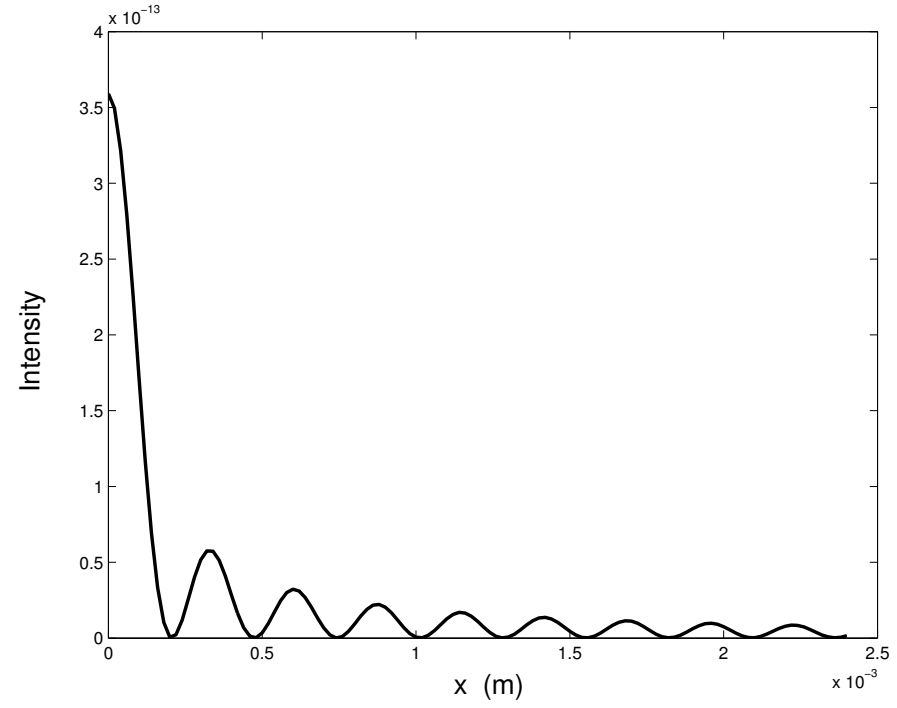

FIG. 7 Intensity profiles predicted in the case of 100 fs pulses using $1^{\text {st }}$-order, $3^{r d}$-order, $5^{\text {th }}$-order Taylor-type developments

case $z_{c}-z_{q}=f_{L_{2}}$ ). The scalar field distribution in the Fourier domain is given by:

$$
\begin{aligned}
\tilde{U}\left(\rho^{\prime}, z^{\prime} ; \omega\right)= & -\frac{i \pi D^{2} \exp \left(i \omega\left(z^{\prime}-z_{q}\right) / c-a\left(z_{c}\right)\right)}{4 \lambda a\left(z_{c}\right)\left(z_{c}-z_{q}\right)} \\
& \times \exp \left(-i \frac{\omega\left(z^{\prime}-z_{c}\right) D^{2}}{8 c\left(z_{c}-z_{q}\right)^{2}}\right) \\
& \times J_{0}\left(\frac{\omega \rho^{\prime} D}{2 c\left(z_{c}-z_{q}\right)}\right) \tilde{G}(\omega)
\end{aligned}
$$

Noting $\beta=\rho^{\prime} D /\left(2 c\left(z_{c}-z_{q}\right)\right)$, we obtain finally after some computation:

$$
\begin{aligned}
I\left(\rho^{\prime}, z^{\prime}\right)= & \frac{C w\left(z_{q}\right)^{4}}{16\left(z_{c}-z_{q}\right)^{2}} \exp \left(-\frac{D^{2}}{2 w\left(z_{q}\right)^{2}}\right) \\
& \times \int_{-\infty}^{+\infty} \frac{1}{1+\tau^{2} \omega^{2}} \exp \left(-\left(\frac{\omega-\omega_{0}}{\Delta \omega}\right)^{2}\right) \\
& \times J_{0}^{2}(\beta \omega) d \omega
\end{aligned}
$$
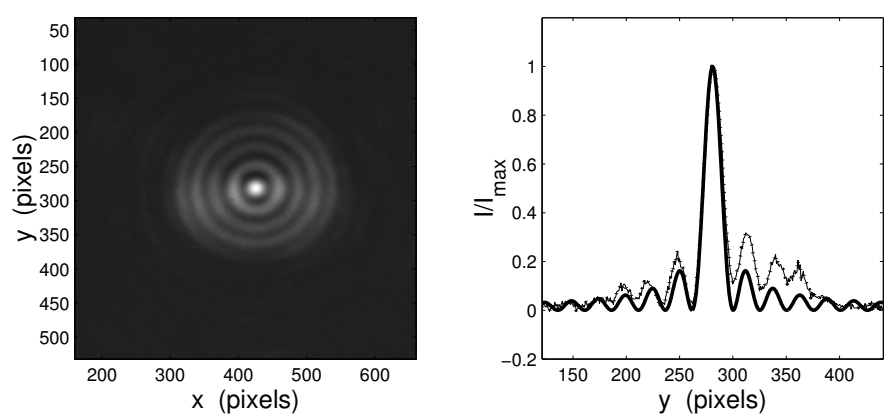

FIG. 8 Beam pattern observed $116 \mathrm{~cm}$ after the lens $L_{2}$ in the case of 100 -fs pulses (left); experimental and theoretical fit using a $1^{\text {st }}$-order Taylor-type development (right) (camera: $11 \mu \mathrm{m}$ per pixel)

This expression is similar to relation (24) replacing $z$ by $z_{c}$, and $\alpha$ by $\beta$. As the typical orders of magnitude of parameters $\alpha$ and 
$\beta$ are quite the same, the previous procedure can be done, and it is easily demonstrated that the field intensity is now given approximately by :

$$
\begin{aligned}
I\left(\rho^{\prime}, z^{\prime}\right)= & \frac{C w\left(z_{q}\right)^{4}}{16\left(z_{c}-z_{q}\right)^{2}} \exp \left(-\frac{D^{2}}{2 w\left(z_{q}\right)^{2}}\right) \\
& \times \frac{\Delta \omega \sqrt{\pi} J_{0}^{2}\left(\beta \omega_{0}\right)}{1+\tau^{2} \omega_{0}^{2}}
\end{aligned}
$$

This expression is of course only valid in the paraxial domain, and for $100 \mathrm{fs}$ pulses whose optical spectrum is not too large. In this domain of validity, expression (29) shows however clearly the diffraction-free nature of the beam because parameter $\beta$ does not depend on the propagation distance after the lens $z^{\prime}$.

Figure 8 shows the experimental pattern observed $116 \mathrm{~cm}$ after the collimating lens, in the case of $100 \mathrm{fs}$ incident pulses emitted by a Ti:Sa laser. The diameter of the opaque disk is $700 \mu \mathrm{m}$. We have $z_{q}=4.5 \mathrm{~cm}$ and $f_{L_{2}}=25 \mathrm{~cm}$. Unfortunately this laser is subject to important astigmatism which disturbs strongly the beam. Beam intensities along the x-axis and $y$-axis are indeed very different and astigmatism is well known to modify Bessel-beam patterns. It is however possible to fit relatively well the experimental beam profile using the approached relation (29). In conclusion, the expression (29) represents a very important simplification of our previous numerical developments [15] which required long calculus times. It allows now the simple optimization of systems.

\section{Conclusion}

Diffraction of a gaussian beam by an opaque disk has been revisited. We could establish analytical expressions that demonstrate that the beam obtained after collimation by a lens can be well approached by a diffraction-free $J_{0}$ Bessel function. We have further demonstrated that a similar analytical expression can be established in the case of femtosecond incident pulses. Those simple analytical relations allow the design of systems in different wavelength regions, while very long computing times were necessary using previous numerical developments. This is a very important feature for us, particularly for further designs of systems in the microwave regime.

\section{References}

[1] J. Durnin, J. J. Miceli Jr, J. H. Eberly “Diffraction-free beams", Phys. Rev. Lett. 58, 1499-1501 (1987)

[2] G. Indebetouw "Nondiffracting optical fields: some remarks on their analysis and synthesis", J. Opt. Soc. Am. A 6, 150-153 (1989)

[3] A. Vasara, J. Turunen, A. T. Friberg "Realization of general nondiffracting beams with computer-generated holograms", J. Opt. Soc. Am. A 6, 1748-1754 (1989)

[4] V. Jarutis, R. Paskauskas, A. Stabinis "Focusing of Laguerre Gaussian beams by axicon", Opt. Commun. 184, 105-112 (2000)
[5] Z. Li, K. Boratay Alici, H. Caglayan, E. Ozbay "Generation of an axially asymmetric Bessel-Like beam from a metallic subwavelength aperture", Phys. Rev. Lett. 102, 143901 (2009)

[6] M. Brunel, S. Coëtmellec "Generation of nondiffracting beams through an opaque disk", J. Opt. Soc. Am. A 24, 3753-3761 (2007)

[7] M. Brunel, D. Mgharaz, S. Coëtmellec "Generation of diffractioncompensated beams with a pigtailed laser diode", IEEE Photonic. Tech. L. 20, 742-744 (2008)

[8] I. A. Litvin, A. Forbes "Bessel Gauss resonator with internal amplitude filter", Opt. Commun. 281, 2385-2392 (2008)

[9] G. Geloni, E. Saldin, E. Schneidmiller, M. Yurkov “Paraxial Green's functions in synchrotron radiation theory", DESY 05-032, ISSN 04189833 (2005)

[10] S.N. Khonina, V. V.Kotlyar, R. V.Skidanov, V. A. Soifer, et.al. “Rotation of microparticles with Bessel beams generated by diffractive elements", J. Mod. Optic. 51, 2167-2184 (2004)

[11] M. A. Porras "Ultrashort pulsed Gaussian light beams", Phys. Rev. E 58, 1086-1093 (1998)

[12] I. P. Christov "Propagation of femtosecond light pulses", 0pt. Commun. 53, 364-366 (1985)

[13] Q. Zou and B. Lu "Propagation properties of ultrashort pulsed beams with constant waist width in free space", opt. Laser Technol. 39, 619-625 (2007)

[14] F. Nicolas, S. Coetmellec, M. Brunel, D. Lebrun "In-line holography with a pulsed-laser beam", Opt. Commun. 268, 27-33 (2006)

[15] M. Brunel, D. Mgharaz, S. Coëtmellec "Ceneration of femtosecond diffraction-compensated beam through an opaque disk", 0pt. Express 16, 10390-10397 (2008) 